\title{
COMBINED CAR-FOLLOWING AND UNSAFE EVENT TRAJECTORY SIMULATION USING AGENT BASED MODELING TECHNIQUES
}

\author{
Montasir M. Abbas \\ Virginia Tech \\ Blacksburg, VA 24061, USA
}

Bryan Higgs

Virginia Tech

Blacksburg, VA 24061, USA

\author{
Linsen Chong \\ MIT \\ Cambridge, MA 02139, USA \\ Alejandra Medina \\ Virginia Tech \\ Blacksburg, VA 24061, USA
}

\begin{abstract}
This paper presents a research effort aimed at modeling normal and safety-critical driving behavior in traffic under naturalistic driving data using agent based modeling techniques. Neuro-fuzzy reinforcement learning was used to train the agents. The developed agents were implemented in the VISSIM simulation platform and were evaluated by comparing the behavior of vehicles with and without agent behavior activation. The results showed very close resemblance of the behavior of agents to driver data.
\end{abstract}

\section{INTRODUCTION}

In the last 50 years, a considerable amount of research has focused on modeling longitudinal driver behavior, producing a large number of car-following models (Treiber et al. 2006), including Gazis-HermanRothery (GHR) models, safety distance models, linear models, and psychophysical or action point models. Most microscopic models assume that human drivers react to the stimuli from neighboring vehicles with the dominant influence originating from the directly leading vehicle. This is known as the "followthe-leader" or "car-following" approximation. Other models introduced "safe time headway" and a desired velocity. Calibrating these car-following models required different levels of effort, and the results depend on data availability, calibration method, and model structure. The GHR model, for instance, requires the calibration of two parameters, limiting the application of the model. The Gipps model(Gipps 1981) (a safe-distance model) uses two different transfer functions for reproducing free-flow and carfollowing conditions. Psychophysical models, on the other hand, assume that the driver will perform an action when a threshold (a function of speed difference and distance) is reached. Estimating distances and speed differences among drivers makes it difficult to calibrate the individual threshold associated with this model. Linear regression and heuristic algorithms (e.g., genetic algorithms) are two widely used methods for model calibration. Despite different mechanisms and software interfaces, when multiple simulation software applications were compared, the resulting behaviors of the models showed similarities (Olstam and Tapani 2004). In addition, it seems that error cannot be eliminated even if more parameters are introduced.

A significant part of the deviations between measured and simulated trajectories can be attributed to the interdriver variability and the intradriver variability (human drivers do not drive constantly over time, and their behavioral driving parameters change). The latter accounts for a large part of the deviations between simulations and empirical observations (Kesting and Treiber 2008). 


\section{Abbas, Chong, Higgs, and Medina}

Table 1 shows the major attributes of the car-following models available in the literature. The multiphase column refers to whether the model calculates the position of the subject vehicle in multiple steps. The multiregime column refers to whether the model calculates the position of the subject vehicle with different equations depending on the regime the subject vehicle falls in.

Table 1: Major similarities and differences between car-following models.

\begin{tabular}{lllllll}
\hline Model & Multiphase & Multiregime & Speed Diff & Space Diff & $\begin{array}{l}\text { Reaction } \\
\text { Time }\end{array}$ & $\begin{array}{l}\text { Variable } \\
\text { Acceleration }\end{array}$ \\
\hline Pipes & Yes & No & Yes & No & Yes & Yes \\
GM & No & No & Yes & Yes & Yes & Yes \\
Wiedemann & No & Yes & Yes & Yes & No & No \\
Fritzsche & No & Yes & Yes & Yes & No & No \\
CA & Yes & No & No & Yes & No & Yes \\
SK & Yes & No & No & Yes & No & Yes \\
IDM & Yes & No & Yes & Yes & Yes & Yes \\
Gipps & Yes & No & Yes & Yes & Yes & Yes \\
VDIFF & No & No & Yes & No & Yes & Yes \\
\hline
\end{tabular}

\section{MODELING VEHICLE TRAJECTORIES}

Several models aim to capture and reconstruct the driver/vehicle driving trajectories. However, little is known about the differences in car-following behavior between driver-vehicle combinations (Ossen and Hoogendoorn 2004). Microsimulation software packages use a variety of car-following models, including Gipps' (AISUM2, SISTM, and DRACULA), Wiedemann's (VISSIM), Pipe's (CORSIM), and Fritzsche's (Paramics). However, parameterization of objective behavior in vehicle trajectory simulation is still in its infancy. Ossen et al.(Ossen and Hoogendoorn 2008) studied the car-following behavior of individual drivers using vehicle trajectory data extracted from high-resolution digital images collected at a high frequency from a helicopter. The analysis was performed by estimating the parameters of different specifications of the GHR car-following rule for individual drivers. The results showed that measurement error has a larger influence than the component of noise. Their study pointed out that optimal parameters differ, and also that the suitability of a car-following model appears to be based on the individual driver data. In addition, Brackstone et al.(Brackstone 2004) used data from a series of instrumented vehicles driven by two groups. The independent variables for this study were age, approximate mileage driven per year, passivity/aggressiveness (P/A, 1-50), driver externality and internality ratings (DE and DI), and Sensation Seeking Scale V (SSSV). Among the results, the study showed that following behavior may be split in two phases (between 30 and 50 miles), and there seems to be an inverse relationship between following distance and sensation seeking. These past efforts, however, did not account for the occasional deviation from normal driving, or the resulting abnormal driving trajectories during safety-critical events.

\section{RESEARCH OBJECTIVES}

The goal of this research was to characterize driver behavior related to driving during normal and critical events, including target speed selection, accelerations, lane changing behavior, car-following distance, response times, and emergency stopping behavior for representative conditions. Of special interest to this research was the integrated modeling of both normal and safety-critical driving beyond existing models' capabilities. Agents were developed to encapsulate individual drivers' decisions in response to various traffic situations as conceptually illustrated in Figure 1. The developed agents were designed and trained to learn individual drivers' actions for any given traffic state. State and action combinations for each individual driver were retrieved from the Virginia Tech Transportation Institute's (VTTI) database of naturalistic driving data. Naturalistic data was collected in a real-world environment, where vehicle speed, acceleration, range, range rate, headway, time to collision (TTC), brake pedal input, were continuously 
recorded over several months for each driver. The resulting large naturalistic database contained both normal and safety-critical events.

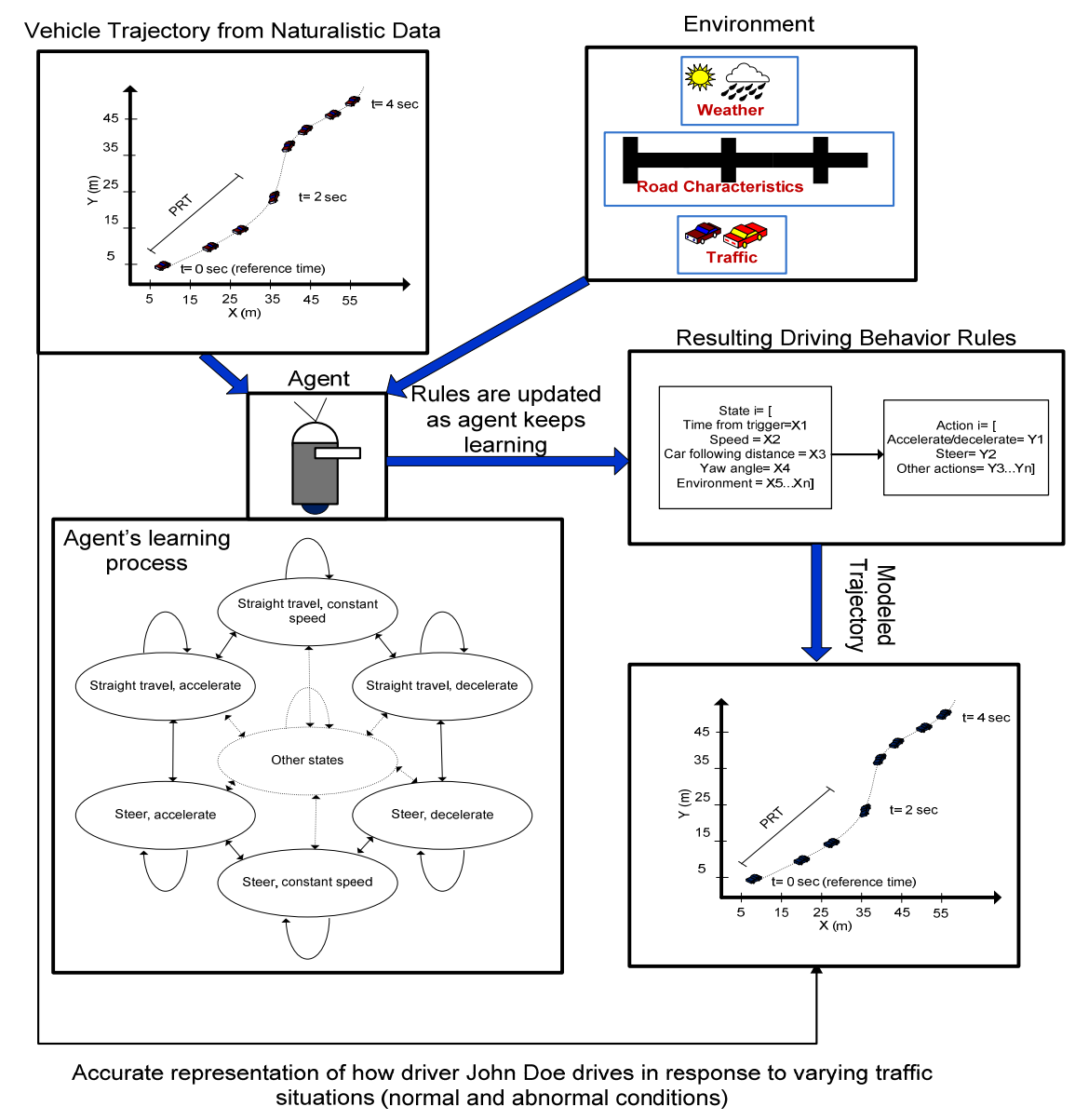

Figure 1: Agent learning process.

\section{TRAINING DATA}

The developed agents were trained using neuro-fuzzy actor-critic reinforcement learning (NFACRL). NFACRL was chosen due to its capability to translate high-traffic state input variables into discrete fuzzy sets and generate continuous action using a weighted average of discrete actions. NFACRL consist of an actor-critic reinforcement learning mechanism that performs a reinforcement learning training through two parts: (1) an actor that is responsible for producing an action corresponding to each state and (2) a critic that is responsible for calculating the long-term reward for the produced action and use the reward to reinforce good actions. The neuro part of the NFACRL mechanism uses a neural network approach to update all the weights responsible for mapping states to actions based on the actor's calculated reward. The fuzzy part of the NFACRL is responsible for transforming the continuous state space into single values representing degrees of membership in predefined discrete fuzzy sets, allowing the algorithm to handle high-dimensional state space (Abbas et al. 2012). All the safety-critical events available from the naturalistic database, and their subsequent normal driving episodes, were used to train 20 agents. The safetycritical events selected in this study are rear-end crash and near-crash conflict, especially when a following vehicle reacts to sudden braking or a sudden merge of the leading vehicle from the adjacent lane. When an event happens, the driver of the following vehicle brakes or swerves to avoid the incoming con- 
Abbas, Chong, Higgs, and Medina

flict. Using naturalistic traffic states and driving actions during crash and near-crash events, this approach was able to reproduce actual driver behavior during normal and safety-critical events with R-squared values as high as 0.98 (Abbas et al. 2011). Cross-validation was performed by applying the training for one agent (driver) to another driver's situation (Figure 2 and Figure 3). The statistical performance of the 20 agents along is shown in Table 2 and Table 3.

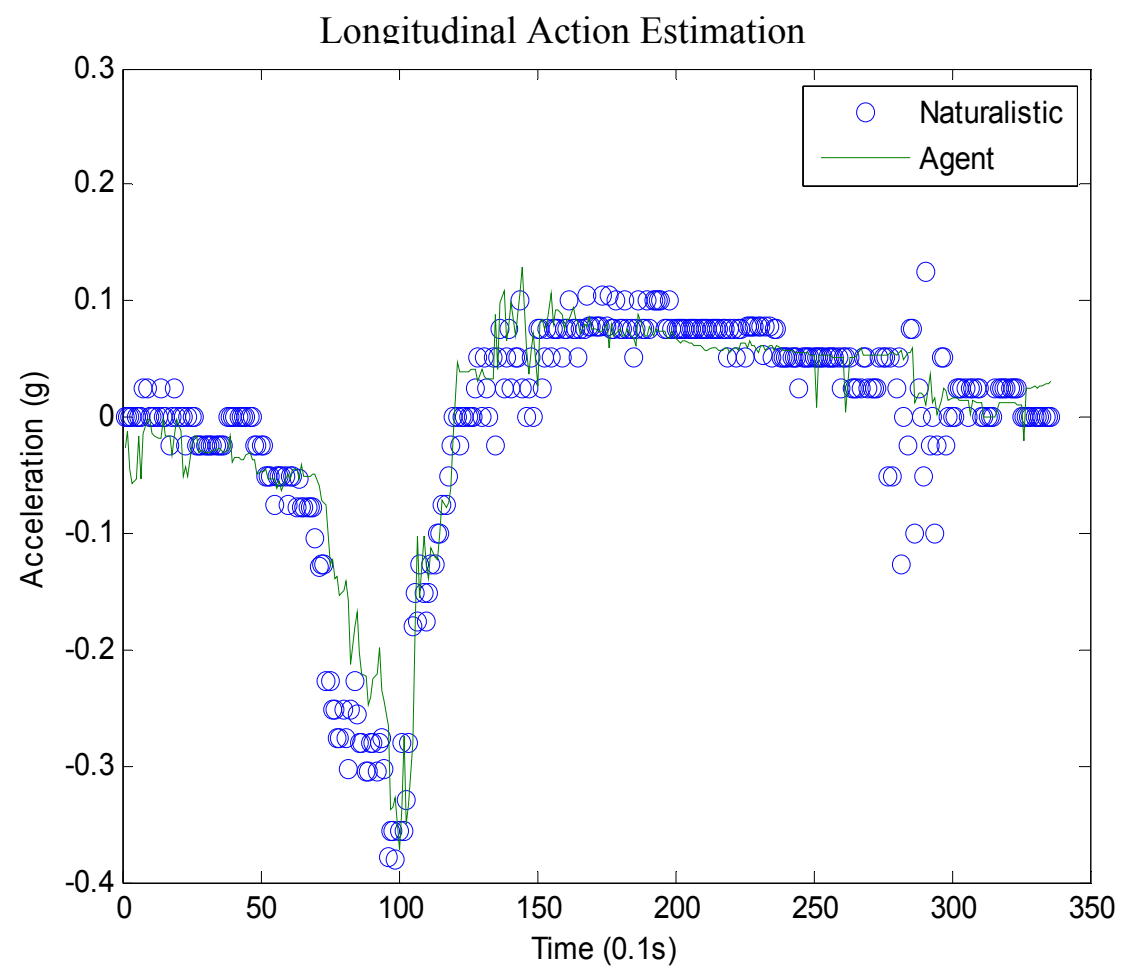

Figure 2: Acceleration of one agent. 
Abbas, Chong, Higgs, and Medina

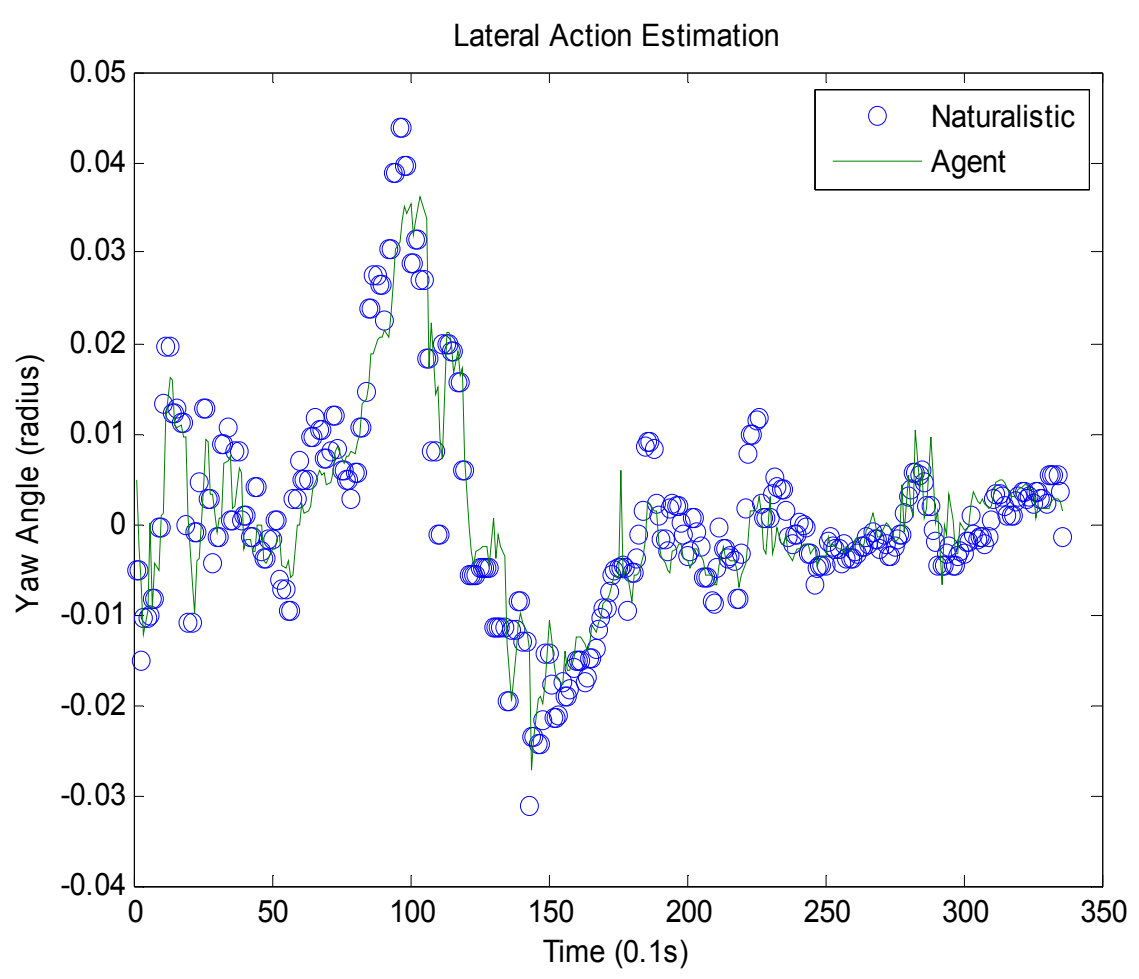

Figure 3: Yaw angle of one agent.

Table 2: R-squared values of the truck agents.

\begin{tabular}{ccccc}
\hline Event & $\begin{array}{c}\text { Agent Longi- } \\
\text { tudinal Ac- } \\
\text { celeration }\end{array}$ & $\begin{array}{c}\text { Agent } \\
\text { Lateral } \\
\text { Accel- } \\
\text { eration }\end{array}$ & $\begin{array}{c}\text { Cross Validation } \\
\text { with Agent A } \\
\text { Longitudinal Ac- } \\
\text { celeration }\end{array}$ & $\begin{array}{c}\text { Cross Val- } \\
\text { idation } \\
\text { with Agent } \\
\text { A Lateral } \\
\text { Accelera- } \\
\text { tion }\end{array}$ \\
\hline Driver A & 0.97 & 0.97 & & 0.97 \\
Driver B & 0.97 & 0.94 & 0.98 & 0.60 \\
Driver C & 0.98 & 0.96 & 0.82 & 0.86 \\
Driver D & 0.99 & 0.92 & 0.86 & 0.64 \\
Driver E & 0.88 & 0.96 & 0.47 & 0.76 \\
Driver F & 0.98 & 0.96 & 0.83 & 0.43 \\
Driver G & 0.86 & 0.98 & 0.86 & 0.62 \\
Driver H & 0.96 & 0.99 & 0.63 & 0.98 \\
Driver I & 0.95 & 0.98 & 0.48 & 0.75 \\
Driver J & 0.85 & 0.98 & 0.66 & 0.32 \\
\hline
\end{tabular}


Abbas, Chong, Higgs, and Medina

Table 3: R-squared values of the car agents.

\begin{tabular}{lcccc}
\hline Event & $\begin{array}{l}\text { Agent Lon- } \\
\text { gitudinal } \\
\text { Acceleration }\end{array}$ & $\begin{array}{l}\text { Agent Lat- } \\
\text { eral Accel- } \\
\text { eration }\end{array}$ & $\begin{array}{l}\text { Cross Vali- } \\
\text { dation with } \\
\text { Agent D } \\
\text { Longitudinal } \\
\text { Acceleration }\end{array}$ & $\begin{array}{l}\text { Cross Vali- } \\
\text { dation with } \\
\text { Agent D } \\
\text { Lateral Ac- } \\
\text { celeration }\end{array}$ \\
\hline Driver a & 0.94 & 0.94 & 0.90 & 0.50 \\
Driver b & 0.97 & 0.94 & 0.82 & 0.30 \\
Driver c & 0.93 & 0.97 & 0.93 & 0.77 \\
Driver d & 0.97 & 0.93 & 0.97 & 0.93 \\
Driver e & 0.95 & 0.98 & 0.68 & 0.95 \\
Driver f & 0.97 & 0.92 & 0.92 & 0.86 \\
Driver g & 0.98 & 0.93 & 0.98 & 0.79 \\
Driver h & 0.98 & 0.93 & 0.98 & 0.90 \\
Driver i & 0.97 & 0.95 & 0.90 & 0.85 \\
Driver j & 0.95 & 0.92 & 0.98 & 0.65 \\
\hline
\end{tabular}

\section{VISSIM IMPLEMENTATION}

The developed agents were implemented into VISSIM simulation platform to allow a combined carfollowing/crash-related trajectory simulation of real life situation. The rationale behind this implementation is shown in Figure 4. The characteristic driving rules of the agents were coded in a microscopic simulation environment (VISSIM) to test and study their effects on agents' behavior in different conditions and scenarios. Snapshots of agent activation parameters are shown in Figure 5 and Figure 6.

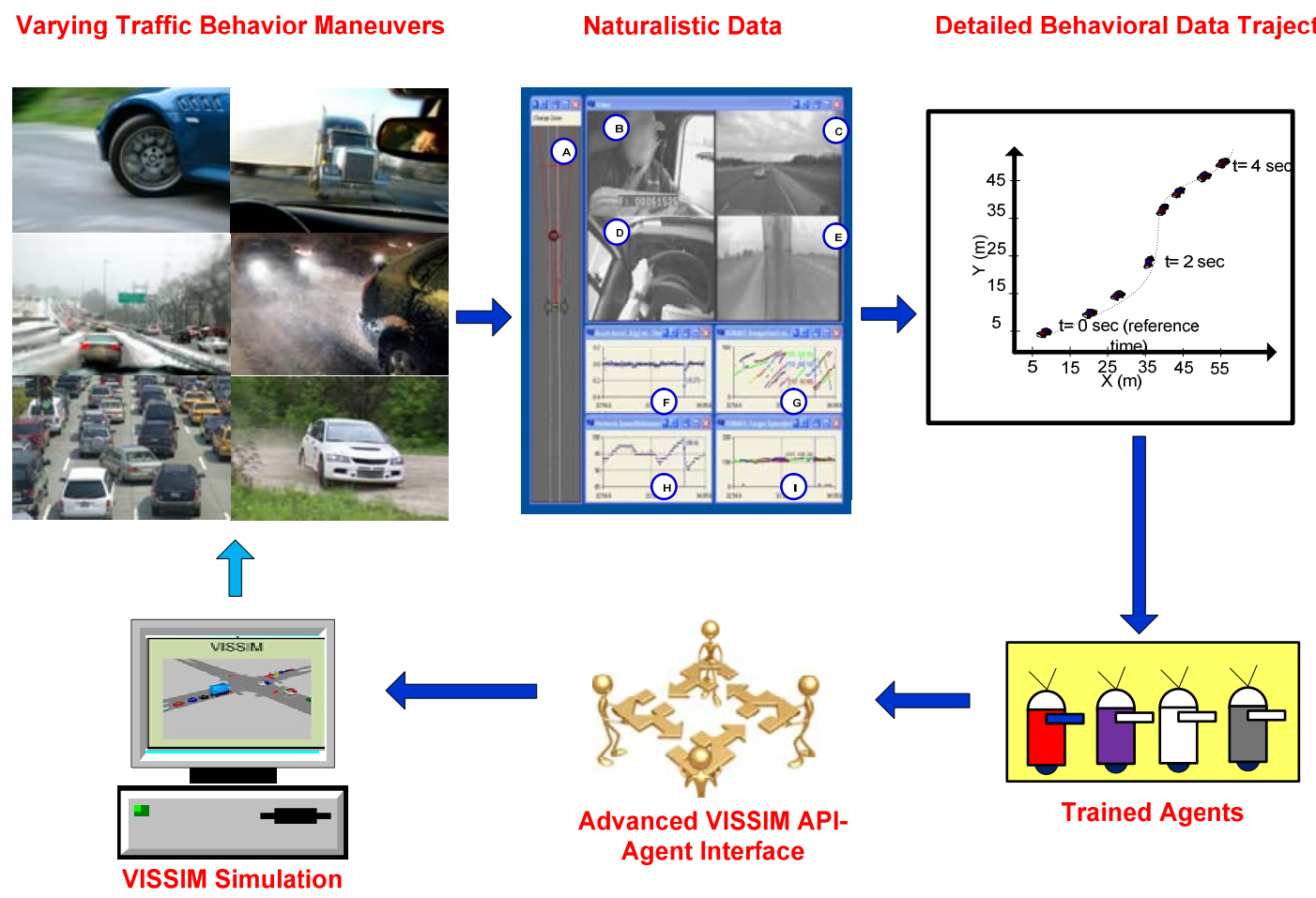

Figure 4: Illustration. System representation of driver's response to encountered traffic situation. 


\section{Abbas, Chong, Higgs, and Medina}

In addition to the model parameters, threshold values are used to define when the driving behavior switches from standard VISSIM (i.e., Wiedemann model) to agent-based behavior and vice versa.

The graphical user interface (GUI) gives access to all user-definable parameters of the agent, as well as to a global threshold value that determines in what situations the agent model should be used instead of the standard VISSIM driving behavior.

To parameterize the agent, the GUI provides tabs for the different model weights and thresholds, as well as a text field for the global thresholds.

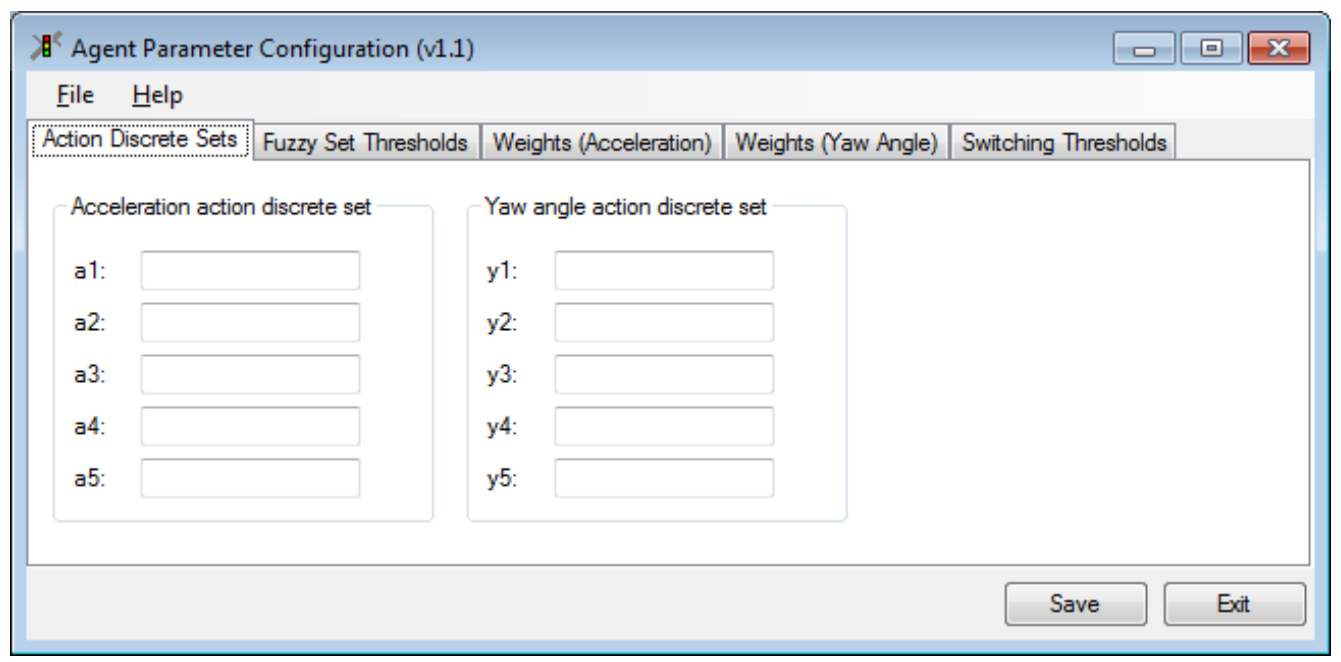

Figure 5: Screen shot. Agent parameter GUI-action discrete sets.

\begin{tabular}{|c|c|c|c|c|c|c|c|}
\hline \multicolumn{5}{|c|}{ 眨 Agent Parameter Configuration (v1.1) } & \multicolumn{3}{|c|}{$\begin{array}{ll} & \square \\
\end{array}$} \\
\hline \multicolumn{8}{|c|}{ File Help } \\
\hline \multicolumn{2}{|c|}{ Action Discrete Sets } & \multirow{2}{*}{$\begin{array}{c}\text { Fuzzy Set Thresholds } \\
\text { a2 (0) }\end{array}$} & \multicolumn{2}{|c|}{ Weights (Acceleration) } & \multirow{2}{*}{$\begin{array}{c}\text { Weights (Yaw Angle) } \\
\mathrm{a} 5(0)\end{array}$} & \multirow[t]{2}{*}{ Switching Thresholds } & \multirow[b]{2}{*}{$\therefore$} \\
\hline Rule & a1 (0) & & $a 3(0)$ & $\mathrm{a} 4(0)$ & & & \\
\hline \multicolumn{8}{|l|}{ • 1} \\
\hline \multicolumn{8}{|l|}{2} \\
\hline \multicolumn{8}{|l|}{3} \\
\hline \multicolumn{8}{|l|}{4} \\
\hline \multicolumn{8}{|l|}{5} \\
\hline \multicolumn{8}{|l|}{6} \\
\hline \multicolumn{8}{|l|}{7} \\
\hline \multicolumn{8}{|l|}{8} \\
\hline \multicolumn{8}{|l|}{9} \\
\hline 10 & & & & & & & - \\
\hline & & & & & & Save & Exit \\
\hline
\end{tabular}

Figure 6: Screen shot. Agent parameter GUI-acceleration weights.

Table 4 shows a description of the parameter file format. The parameter file is a comma-separated text file, and all numbers are double precision format. 
Table 4: Parameter file description.

\begin{tabular}{ll}
\hline Description & Values (in blocks, separated by commas); see APC documentation \\
\hline $\begin{array}{l}\text { Fuzzy Set Layer Thresh- } \\
\text { olds }\end{array}$ & Fuzzy Set Layer Thresholds \\
thresholdSpeed: & lowerthresholdSpeed, upperthresholdSpeed \\
thresholdRange: & lowerthresholdRange, upperthresholdRange \\
thresholdRangeRate: & lowerthresholdRangeRate, upperthresholdRangeRate \\
thresholdAcceleration: & lowerthresholdAcceleration, upperthresholdAcceleration \\
thresholdLaneAngle: & lower thresholdLaneAngle, upper thresholdLaneAngle \\
thresholdOffset: & lower thresholdLaneAngle, upper thresholdLaneAngle \\
AccelerationDiscreteSet: & a1,a2,a3,a4,a5 \\
LaneAngleDiscreteSet: & la2,la2,la3,la4,la5 \\
Weights W1(i,j): & W1 $(1,1), \ldots$, W1 $(1,5)$, W1 $(2,1), \ldots$, W1(i,j),...,W1(32,5) \\
Weights W2(1,j): & W2 $(1,1), \ldots$, W2 $(1,5)$, W2 $(2,1), \ldots$, W2(i,j),...,W2(32,5) \\
Switch Conditions & There will be one (or more) threshold value that determines if the agent \\
& model is switched on or off. At the moment, this is Time to Collision. It \\
& can, of course, be changed to other values. \\
\hline
\end{tabular}

Finally, Figure 7 shows an example VISSIM simulation/animation of a safety-critical behavior that represents a run-off-the-road incident where a vehicle is simulated to divert from the road network. The simulation of this behavior in VISSIM environment would have not been possible without this approach.

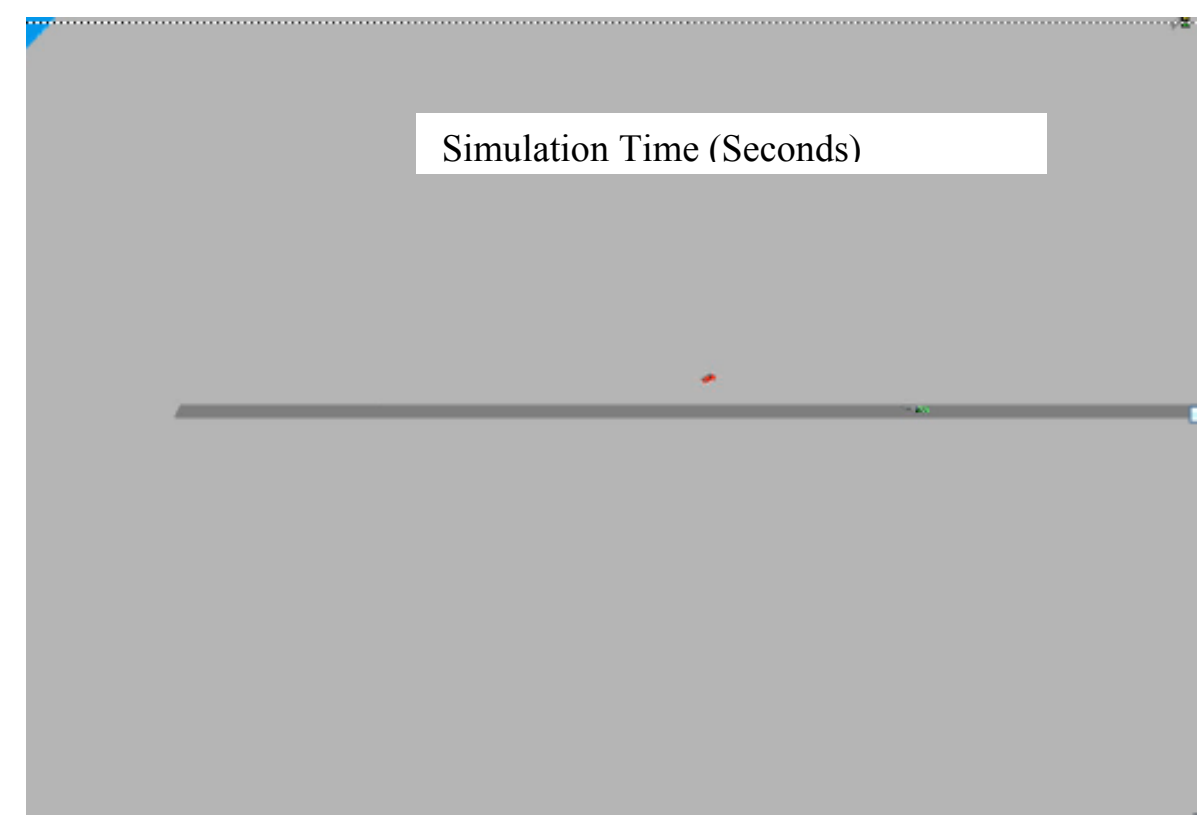

Figure 7: A simulation of a run-off-the-road incident.

\section{CONCLUSIONS}

One of the major contributions of this research is the developed integrated framework for safety and operation analysis. An artificial intelligence machine-learning technique was developed and used to model driving behavior during normal and safety-critical events. Naturalistic driving database was used to train and validate driver agents. The proposed methodology also simulated events from different drivers and 


\section{Abbas, Chong, Higgs, and Medina}

proved behavior heterogeneities. The developed agents were implemented in the VISSIM simulation platform and were evaluated by comparing the behavior of vehicles with and without agent activation. The results showed very close resemblance of the behavior of agents and driver data. This research is expected to provide the simulation and modeling industry with methods for developing more accurate and more sensitive traffic models. It could also enable future research to develop new traffic simulation models that accurately model driver behavior during incidents and other complex traffic situations.

\section{ACKNOWLEDGMENTS}

The research presented in this paper was a collaborative effort among several agencies and individuals at Virginia Tech, Virginia Center for Transportation Innovation and Research (VCTIR), and PTV. Researchers who contributed to the study are: Cathy McGhee, Mike Fontain, Noah Goodall, Lukas Kautzsch, Axel Leonhardt, and Kiel Ova. The project was supported by the Federal Highway Administration under Agreement No. DTFH61-09-H-00007. Any opinions, findings, and conclusions or recommendations expressed in this publication are those of the Author(s) and do not necessarily reflect the view of the Federal Highway Administration.

The authors would like to express thanks to Dr. C.Y. David Yang, the FHWA Agreement Manager, for his continued support and guidance during this project.

\section{REFERENCES}

Abbas, M., et al. 2012. Agent-Based Reinforcement Learning Model for Simulating Driver Heterogeneous Behavior During Safety-Critical Events in Traffic. Proceedings of the Transportation Research Board Annual Meeting.

Abbas, M., et al. 2011. Agent-based Evaluation of Driver Heterogeneous Behavior during Safety Critical Events. 14th International IEEE Conference on Intelligent Transportation Systems, ITSC '11.

Brackstone, M. 2004. Driver Psychological Types and Car Following:Is There a Correlation? Results of a Pilot Study.

Gipps, P. G. 1981. "A Behavioural Car-Following Model for Computer Simulation." Transportation Research Board 15B(2-C): 7.

Kesting, A. and M. Treiber. 2008. "Calibrating Car-Following Models using Trajectory Data: Methodological Study." 17.

Olstam, J. J. and A. Tapani. 2004. Comparison of Car-following models, VTI Meddelande 990A, Swedish National Road Transport Research Institute. Project Code 40503 and 40485, 2004.

Ossen, S. and S. P. Hoogendoorn. 2004. Car-following Behavior Analysis from Microscopic Trajectory Data.

Ossen, S. and S. P. Hoogendoorn. 2008. "Validity of trajectory-based calibration approach of carfollowing models in presence of measurement errors." Transportation Research Record(2088): 117125.

Treiber, M., et al. 2006. "Delays, Inaccuracies and Anticipation in Microscopic Traffic Models." 28.

\section{AUTHOR BIOGRAPHIES}

MONTASIR ABBAS is an Associate Professor at Virginia Tech. He received his Ph.D. in Civil Engineering from Purdue University in 2001. He has previously worked as an Assistant Research Engineer at Texas Transportation Institute and as a Visiting Assistant Professor at Texas A\&M. His e-mail is abbas@vt.edu.

LINSEN CHONG is a Research Assistant at MIT. He received his MS in Civil Engineering from Virginia Tech in 2011. His e-mail is linsenc@mit.edu. 
Abbas, Chong, Higgs, and Medina

BRYAN HIGGS is a Research Assistant at Virginia Tech. He received his MS in Civil Engineering from Virginia Tech in 2011. His e-mail iswuhbaam@vt.edu.

ALEJANDRA MEDINA is a Senior Research Associate at Virginia Tech Transportation Institute. She received her MS in Civil Engineering from Arizona State University in 1995. Her e-mail is ale@vtti.vt.edu. 